\title{
"Exhaustive" Baeyer-Villiger Oxidation of Poly(Methyl Vinyl Ketone) and Its Copolymers
}

\author{
Pengfei Ma, ${ }^{[a]}$ Christopher M. Plummer, ${ }^{[b]}$ Wenjun Luo, ${ }^{[a]}$ Jiyan Pang, ${ }^{[c]}$ Yongming Chen, ${ }^{[\mathrm{d}]}$ and Le $\mathrm{Li}^{*[\mathrm{a}]}$
}

\begin{abstract}
The development of "exhaustive" (nearly quantitative) post-modification reactions relies heavily on the efficiency of their corresponding small-molecule protocols. However, the direct translation of existing small-molecule protocols into postpolymerization modifications were often troublesome due to undesired side reactions. For example, the development of an "exhaustive" Baeyer-Villiger (BV) post-modification using existing small-molecule BV protocols suffered from a lack of reactivity or significant chain scission. Herein, we demonstrate that a careful reoptimization of a small-molecule transformation on a polymer substrate allowed us to overcome such challenges, thereby enabling an "exhaustive" BV post-polymerization modification. Furthermore, a one-pot copolymerization/"exhaustive" BV post-modification procedure was developed to produce copolymers of vinyl acetate (VAc) and more activated monomers (MAMs) in a convenient and scalable manner. This user-friendly methodology provides a general access to synthetically challenging poly(VAc-co-MAM)s including both statistical and narrow-dispersed block copolymers and could greatly facilitate the exploration of applications with such materials.
\end{abstract}

\section{Introduction}

Complementary to conventional polymerization approaches, post-polymerization modification represents another appealing strategy for polymer synthesis. ${ }^{[1]}$ Like organic chemists striving for highly efficient small-molecule transformations, polymer chemists seek "ideal" post-polymerization modifications where an "exhaustive" (nearly quantitative) functionalization can be reached. However, only a few types of transformations meet the standard of "exhaustive" post-modification. The most notable examples involve "click" reactions ${ }^{[2]}$ and multicomponent reactions. ${ }^{[1 c, 3]}$ Indeed, the development of an "ideal" or "exhaustive" post-polymerization modification reaction is even more technically challenging than that of its small-molecule prototype (Scheme 1A and Figure S1). As such, any deviation from an "ideal" post-modification will be significantly magnified with an increase in the degree of polymerization (DP) of the polymer, thereby making the post-modification of polymers with high

\footnotetext{
[a] P. Ma, W. Luo, Prof. L. Li*

PCFM Lab and GDHPRC Lab, School of Chemistry, Sun Yat-sen University, Guangzhou 510275, P. R. China; E-mail: lile26@mail.sysu.edu.cn

[b] Dr. C. M. Plummer

Aix-Marseille University, CNRS, Institut de Chimie Radicalaire, UMR 7273, Marseille

[c] Prof. J. Pang

School of Chemistry, Sun Yat-sen University, Guangzhou 510275 P. R. China

[d] Prof. Y. Chen

PCFM Lab and GDHPRC Lab, School of Materials Science, Sun

Yat-sen University, Guangzhou 510275, P. R. China
}

molecular weight (MW) particularly difficult. An "exhaustive" post-polymerization modification reaction requires both a high degree of mechanistic fidelity and sufficient functionalization rates to prevent the production of defects, ${ }^{[4]}$ chain scission or cross-linking, ${ }^{[1 b, 5]}$ since any side reaction will negatively alter the properties of the resultant polymers. Such stringent requirements greatly limit the reaction diversity ${ }^{[6]}$ of "exhaustive" postpolymerization modification methods. Accordingly, many mechanistically complex reactions such as oxidations, which can induce unpredictable decomposition, have been less studied in the realm of post-polymerization modification. ${ }^{[7]}$ Recently, Klausen ${ }^{[8]}$ and Ouchi ${ }^{[9]}$ individually developed "exhaustive" oxidative postmodifications for their boron-containing polymers. Owing to the high efficiency of organoboron oxidation chemistry, ${ }^{[10]} \mathrm{C}-\mathrm{B}$ bonds on their tailor-made polymers were "nearly quantitatively" cleaved by hydrogen peroxide to form free hydroxyl groups after hydrolysis. Encouraged by these accomplishments, we aimed to challenge if an "exhaustive" oxidative post-polymerization modification could occur at relatively inert $\mathrm{C}-\mathrm{C}$ bonds instead of $\mathrm{C}-\mathrm{B}$ bonds. We were particularly interested in developing an "exhaustive" Baeyer-Villiger (BV) oxidation of poly(methyl vinyl ketone) and its copolymers for post-polymerization modification. Such a transformation may provide a general access to synthetically challenging copolymers of vinyl acetate (VAc) and more activated monomers (MAMs), whose reactivities are mismatched in copolymerization (Scheme 1B). ${ }^{[11,12]}$ In this case, commercially available methyl vinyl ketone (MVK) would be used as a "masked-VAc" monomer since MVK readily copolymerizes with other MAMs ${ }^{[11,12]}$ and is also compatible with multiple reversible deactivation radical polymerization (RDRP) techniques. ${ }^{[13]}$

Although the translation of small-molecule BV oxidation ${ }^{[14]}$ into post-polymerization modification seemed promising, previous unrewarding studies ${ }^{[15]}$ in the literature suggested that significant challenges might still exist. Indeed, just like other oxidative transformation, multiple side-reaction pathways such as aldol reaction/condensation, ${ }^{[16]}$ undesired oxidation ${ }^{[17]}$ and radical cleavage ${ }^{[18]}$ are known to be associated with BV oxidation. Such side reactions could introduce defects into the polymer backbone and negatively affect the MW and dispersity $(\Theta)$ of polymers. In addition, uncertainty regarding the regioselectivity of the proposed BV post-modification has to be considered since such selectivity for a polymer substrate has never been established.

Herein, we present an "exhaustive" BV post-modification method that is able to prepare a wide range of homopolymers, statistical, and block copolymers of VAc from commercially available poly (methyl vinyl ketone) (PMVK) and various PMVK copolymers. The efficiency of this method ensures that almost every ketone site on the PMVK segment is converted into an ester while the MW and $\oslash$ of polymers remain essentially unchanged during oxidation. 


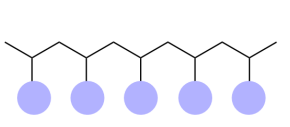

Starting Polymers

(SPs)
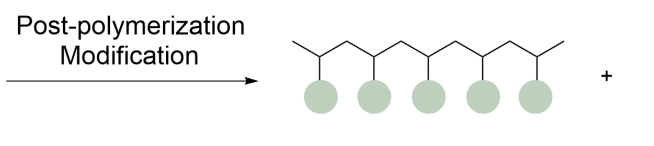

"Ideal"

Functionalized polymers (IFPs)

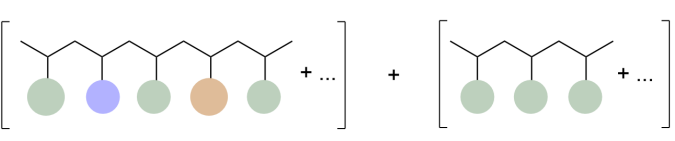

Functionalized Polymers with Defects
Functionalized Polymers with Chain Scissions

\section{B. A General Approach to Access Synthetically Challenging Poly(VAc-co-MAM)s}

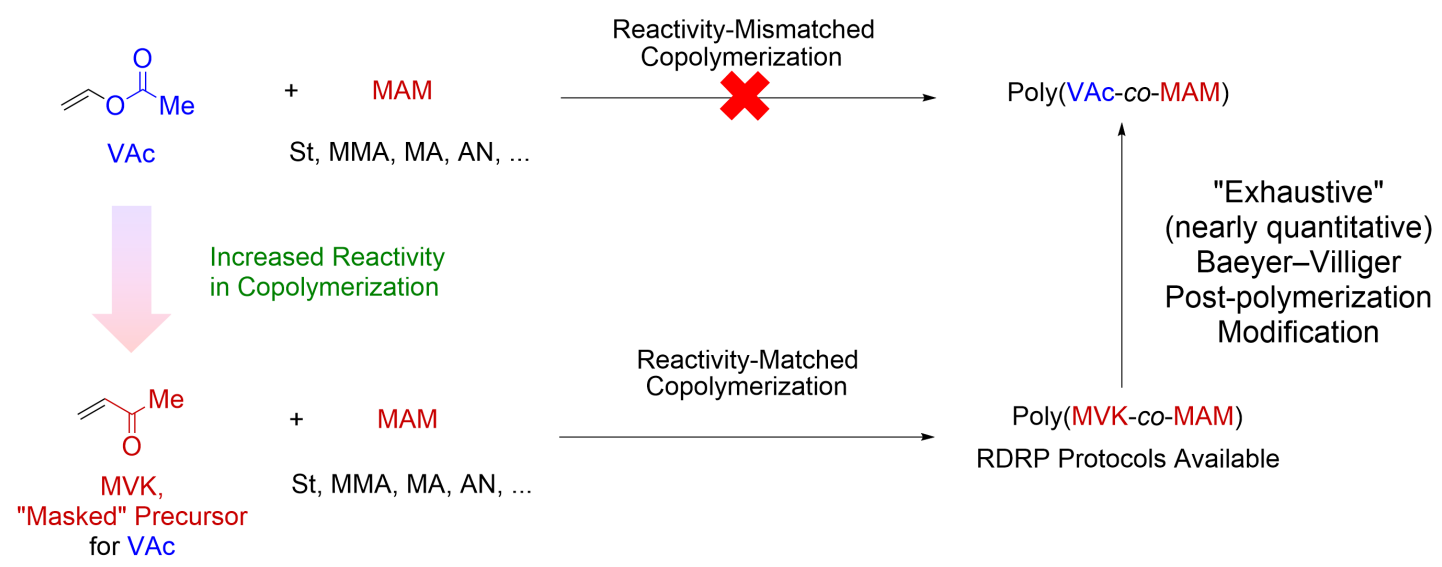

Scheme 1. Accessing synthetically challenging Poly(VAc-co-MAM)s with an "exhaustive" Baeyer-Villiger post-polymerization modification.

\section{Results and Discussion}

Initial Attempts Using Classical BV Protocols. Pioneering efforts ${ }^{[15]}$ to bring BV oxidation into the field of postpolymerization modification suffered from a lack of reactivity and/or significant chain scission. Conventional BV oxidation conditions $^{[14]}$ appeared to be destructive and incompatible with ketone-containing polymers. To obtain more mechanistic details, we decided to re-investigate this transformation using PMVK as a substrate. ${ }^{[19]}$ We first evaluated the influence of temperature and reaction time using a commercial PMVK sample (Figure 1 and Table S2). The BV oxidations of PMVK were conducted using a typical protocol of chloroform as a solvent and $m$ chloroperoxybenzoic acid ( $m$ CPBA) as an oxidant. Although higher conversions were obtained with prolonged periods at elevated temperatures, the MW of the polymers decreased significantly. Noticeably, our kinetic studies revealed that the BV post-modification proceeded rapidly during the early stages, but became sluggish after $24 \mathrm{~h}$. Consistent with the early investigations, ${ }^{[15]}$ our results also suggested that the standard BV oxidation conditions were not efficient enough to complete the proposed post-modification of PMVK.

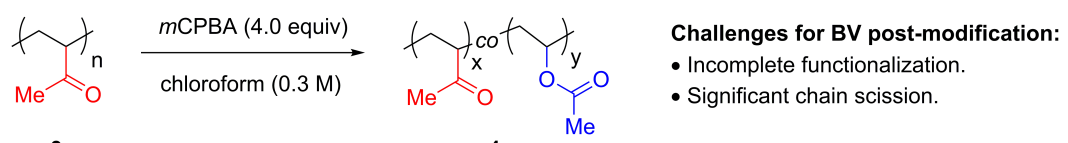

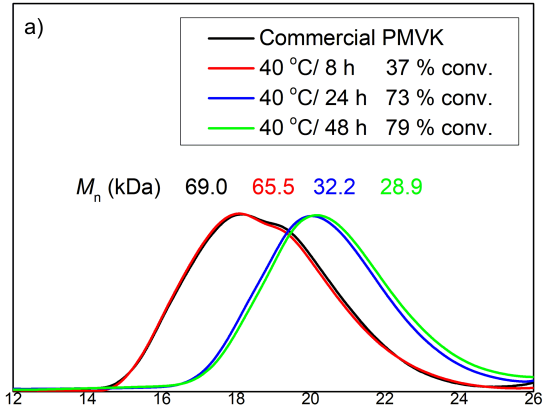

Elution time $(\mathrm{min})$

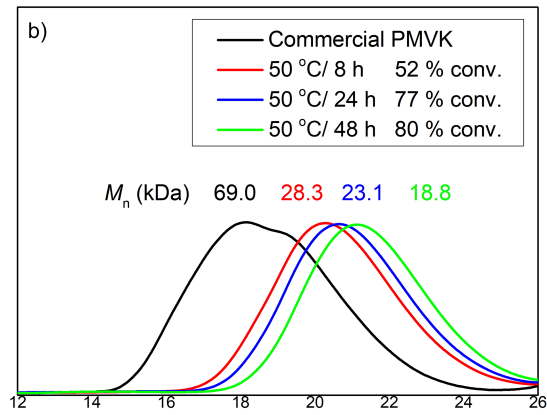

Elution time ( $\mathrm{min})$

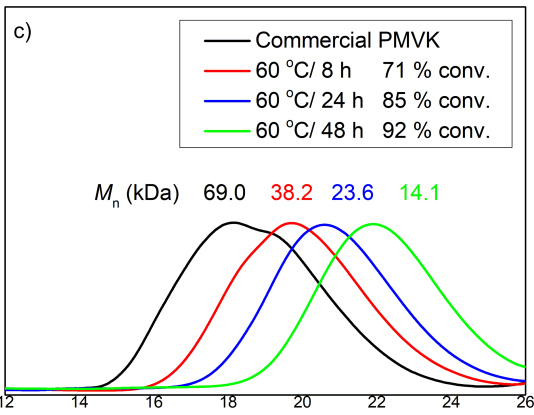

Elution time ( $\mathrm{min})$

Figure 1. The influence of temperature and reaction time on the BV oxidation of PMVK in chloroform. SEC traces of commercial PMVK $\mathbf{3 a}$ before and after BV oxidation at (a) $40^{\circ} \mathrm{C}$, (b) $50^{\circ} \mathrm{C}$ and (c) $60^{\circ} \mathrm{C}$ with different reaction time. 

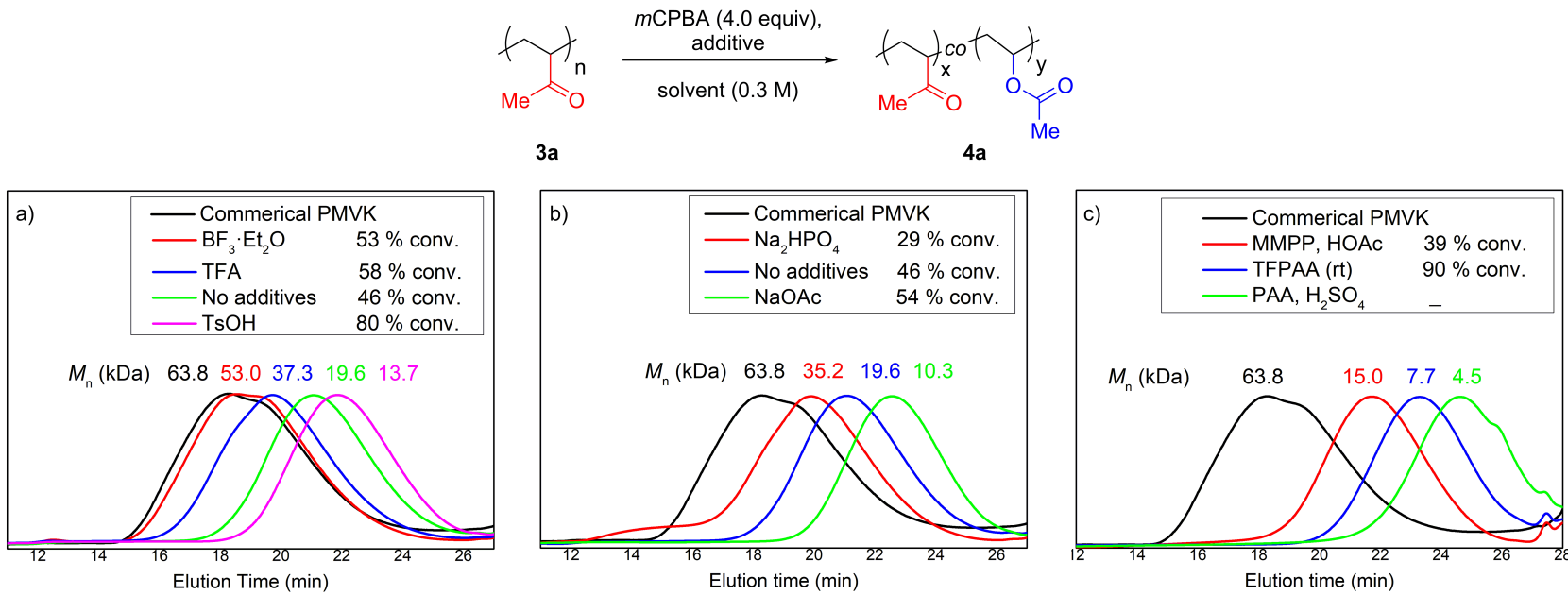

Figure 2. BV oxidation of PMVKs using high-yielding conditions optimized for the small-molecule model. SEC traces before and after BV oxidations: (a) acid catalyst, $m$ CPBA as oxidant, (b) in the presence of inorganic base, $m$ CPBA as oxidant, (c) MMPP and other peroxyacids as oxidant. (See the detailed conditions in Table S3.)

Unsuccessful Attempts at the Translation of Small-molecule Protocols to PMVK. A small-molecule model using 3hexylundecan-2-one as a substrate was established at the outset of this study. A large number of high-yielding conditions from the literature were screened and modified (Table S1). Unfortunately, the direct translation of small-molecule protocols to the post-modification of PMVK completely failed (Figure 2 and Table S3). The $M_{\mathrm{n}}$ of the polymers decreased significantly with the use of either acids or bases (Figure $2 a$ and $b$ ). In addition, the use of alternative oxidants such as peracetic acid (PAA), trifluoroperoxyacetic acid (TFPAA), and magnesium monoperoxyphthalate hexahydrate (MMPP) gave even worse results (Figure 2c).

The failure of the translation of the small-molecule model to PMVK suggests that the reactivity of a molecule with a single functional group such as 3-hexylundecan-2-one is quite different from that of PMVK. For a polymeric substrate, the electronic and steric interactions between functional groups will significantly affect its reactivity and possibly induce additional side reactions. Therefore, a small-molecule model would not always be able to guide the development of synthetic methods for polymers and other macromolecules.

Accordingly, we turned our focus to PMVK instead of the small-molecule model compound in the following studies. Based on the estimation in Figure S1, the MW of a polymer with DP = 1000 will be cut in half even if only $0.1 \%$ chain scission were to occur during post-modification. Therefore, the suppression of side reaction pathways was considered highly important for the post-modification reaction. A literature survey revealed multiple side reaction pathways for BV oxidation. For example, acidinduced radical generation ${ }^{[18]}$ which is reported to be able to initiate even at low temperatures could cause significant chain

Table 1. The effect of solvent on the BV oxidation of commercial PMVK $3 a^{[a]}$

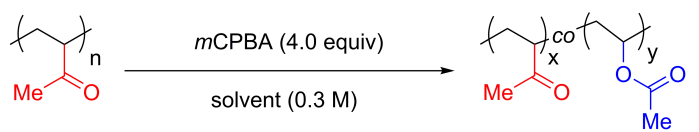

3a

\begin{tabular}{|c|c|c|c|c|c|c|c|c|c|}
\hline Entry & Solvent & $\begin{array}{c}\text { Conversion }^{[b]} \\
(\%)\end{array}$ & $\begin{array}{c}M_{\mathrm{n}, \mathrm{SEC}}{ }^{[\mathrm{c}]} \\
(\mathrm{kDa})\end{array}$ & $\oplus^{[\mathrm{c}]}$ & Entry & Solvent & $\begin{array}{c}\text { Conversion }^{[\mathrm{b}]} \\
(\%)\end{array}$ & $\begin{array}{c}M_{\mathrm{n}, \mathrm{SEC}}{ }^{[\mathrm{c}]} \\
(\mathrm{kDa})\end{array}$ & $\bigoplus^{[c]}$ \\
\hline 1 & $\mathrm{CHCl}_{3}$ & 55 & 36.1 & 2.18 & 12 & DMF & 11 & 60.8 & 2.53 \\
\hline 2 & $\mathrm{CH}_{2} \mathrm{Cl}_{2}$ & 44 & 41.9 & 2.27 & 13 & $n$-hexane & 86 & 64.0 & 2.43 \\
\hline 3 & 1,2-dichloroethane & 52 & 61.9 & 2.50 & 14 & toluene & 34 & 63.6 & 2.61 \\
\hline 4 & EtOAc & 23 & 63.9 & 2.45 & 15 & (trifluoromethyl)benzene & 80 & 63.7 & 2.44 \\
\hline 5 & THF & 14 & 60.8 & 2.58 & 16 & fluorobenzene & 71 & 69.8 & 2.61 \\
\hline 6 & $\mathrm{Et}_{2} \mathrm{O}$ & 26 & 63.3 & 2.55 & 17 & chlorobenzene & 75 & 71.3 & 2.57 \\
\hline 7 & dioxane & 12 & 62.1 & 2.56 & 18 & m-dichlorobenzene & 79 & 67.7 & 2.49 \\
\hline 8 & $\mathrm{EtOH}$ & 22 & 60.8 & 2.53 & 19 & o-dichlorobenzene & 81 & 65.4 & 2.46 \\
\hline 9 & $\left(\mathrm{CF}_{3}\right)_{2} \mathrm{CHOH}$ & 16 & 55.2 & 2.49 & 20 & 1,2,4-trichlorobenzene & 85 & 68.5 & 2.38 \\
\hline 10 & $\mathrm{MeCN}$ & 16 & 54.1 & 2.43 & 21 & 1,2,4-trichlorobenzene ${ }^{[\mathrm{d}]}$ & $>99$ & 49.4 & 2.29 \\
\hline 11 & $N$-methylpyrrolidone & 12 & 59.6 & 2.58 & 22 & 1,2,4-trichlorobenzene $\mathrm{e}^{[\mathrm{d}, \mathrm{e}]}$ & $>99$ & 66.4 & 2.42 \\
\hline
\end{tabular}

[a] Standard conditions: PMVK 3a (41.7 mg, containing $0.60 \mathrm{mmol}$ repeating units), $\mathrm{mCPBA}(2.40 \mathrm{mmol})$, and solvent $(2.00 \mathrm{~mL})$ stirred at $50{ }^{\circ} \mathrm{C}$ for 8 h. The $M \mathrm{n} / \oplus$ of PMVK was $66.0 \mathrm{kDa} / 2.54$. [b] Determined by ${ }^{1} \mathrm{H}$ NMR of the crude products using 1,1,1,3-tetrachloropropane as an internal standard. [c] Determined by SEC (THF) analysis relative to polystyrene standards. [d] Reaction was run for $24 \mathrm{~h}$ instead of $8 \mathrm{~h}$. The $M_{\mathrm{n}} / \oplus$ of PMVK was 61.4 $\mathrm{kDa} / 2.54$. [e] Hexamethyldisiloxane $(1.20 \mathrm{mmol})$ and dry $m$ CPBA were employed. 


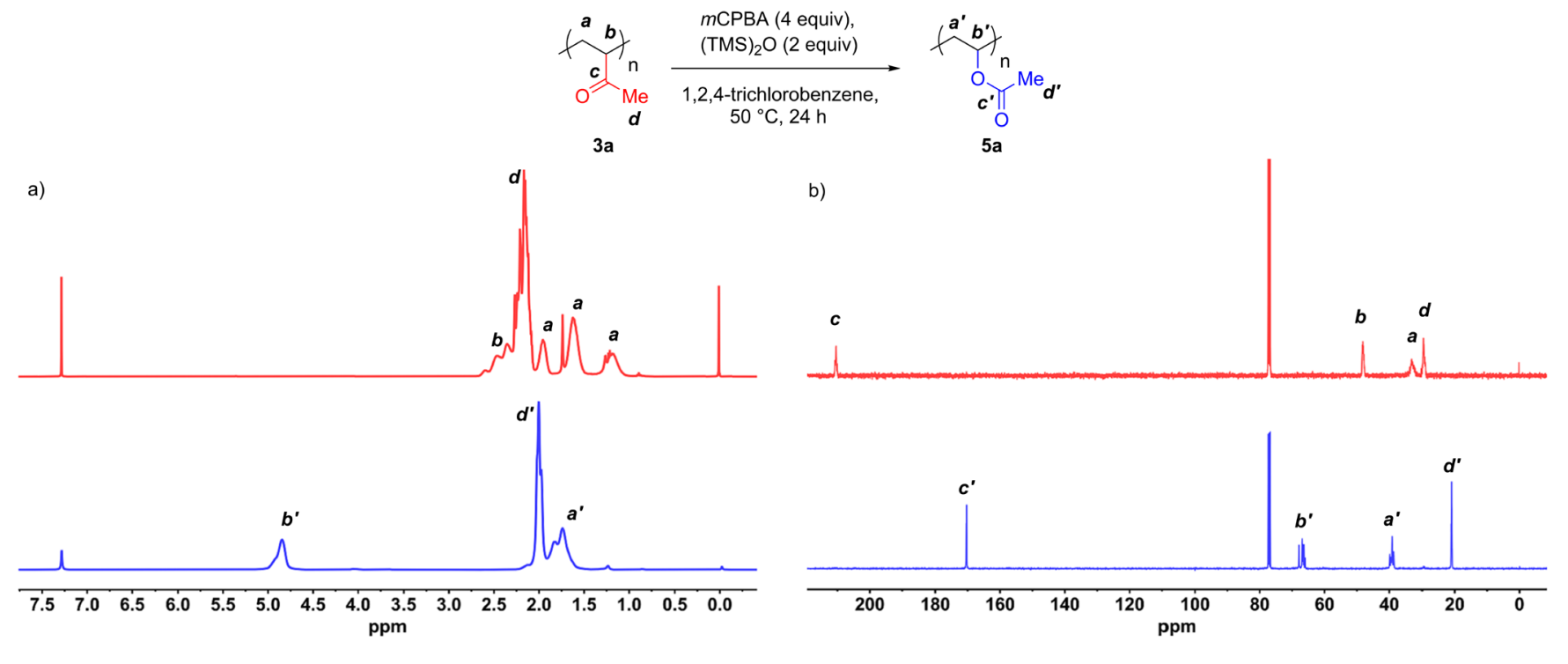

Figure 3. "Exhaustive" BV oxidation of commercial PMVK homopolymers. Comparison of (a) ${ }^{1} \mathrm{H}$ NMR spectra and (b) ${ }^{13} \mathrm{C}$ NMR spectra before and after "exhaustive" BV oxidation of commercial PMVK 3a. The NMR spectra were recorded using $\mathrm{CDCl}_{3}$ as a solvent.

scission. In addition, oxidative decomposition ${ }^{[17]}$ instigated by excessive oxidants, or Aldol-type condensations ${ }^{[16]}$ promoted by acid or base could also complicate the post-polymerization modification. A combination of a literature survey and our preliminary results suggested that a comprehensive evaluation of PMVK under BV conditions was required.

Development of the BV Oxidation of Commercial PMVK Homopolymers. We first identified that the combination of carbon-13 and proton nuclear magnetic resonance spectroscopy $\left({ }^{13} \mathrm{C}\right.$ and ${ }^{1} \mathrm{H}$ NMR) was a reliable method to quantitatively characterize both PMVK and its product after BV oxidation. Errors relating to $\mathrm{C}-\mathrm{H}$ decoupling of ${ }^{13} \mathrm{C}$ NMR were evaluated and calibrated by ${ }^{1} \mathrm{H}$ NMR prior to use.

The effect of solvent was first investigated under a set of standard conditions (Table 1). Less polar solvents generally gave higher conversions while polar solvents were found to be problematic. Dichloromethane and chloroform, two common solvents for BV oxidation, were not suitable for post-polymerization modification due to both significant polymer degradation and only moderate reaction rates. Surprisingly, the optimal solvents were found to be halogen-substituted aromatic solvents such as fluorobenzene, chlorobenzene and 1,2,4-trichlorobenzene, which are rarely used in BV oxidation. Their improved performance may be partially attributed to the enhanced stability of these solvents to oxidative conditions. Among the solvents tested, 1,2,4-trichlorobenzene was found to be optimal in terms of both conversion and $M_{\mathrm{n}}$. The post-polymerization modification proceeded smoothly in the first $8 \mathrm{~h}$, but the $M_{\mathrm{n}}$ of the functionalized polymers declined with prolonged periods. We conjectured that the accumulation of $m$-chlorobenzoic acid was likely the cause of this phenomenon.

We speculated that a "precise" buffer could perhaps maintain the $\mathrm{pH}$ of the reaction mixture within a narrow window where polymer degradation would not occur. Indeed, even a subtle deviation from the "ideal" $\mathrm{pH}$ range could potentially induce chain scission. Initial efforts using various aqueous buffers were unsuccessful. Control experiments indicated that excessive water affected both the reaction rate and $\mathrm{MW}$ in a negative manner.
Gratifyingly, an "exhaustive" screening (>700 experiments) of inorganic salts and organic additives revealed that hexamethyldisiloxane (TMS) ${ }_{2} \mathrm{O}$ (Table S5-S7) was able to buffer the system effectively. We conjectured that (TMS $)_{2} \mathrm{O}$ slowly reacted with the $m$-chlorobenzoic acid accumulating during the oxidation to form a neutral trimethylsilyl $m$-chlorobenzoate species. Further suppression of chain scission by drying the commercial $m$ CPBA reagent provided an optimal BV protocol for the "exhaustive" functionalization of PMVK.

"Exhaustive" BV Oxidation of Commercial PMVK Homopolymers. With the first "exhaustive" functionalization condition in hand, we conducted a BV oxidation of commercial PMVK polymers on a preparative scale. In addition to ${ }^{1} \mathrm{H}$ NMR and ${ }^{13} \mathrm{C}$ NMR, the resulting polymer was fully characterized by size exclusion chromatography (SEC), Fourier transform infrared spectroscopy (FT-IR), differential scanning calorimetry (DSC), and thermogravimetric analysis (TGA).

The ${ }^{1} \mathrm{H}$ NMR and ${ }^{13} \mathrm{C}$ NMR spectra of the polymer samples before and after oxidation are displayed in Figure 3 . The peak at $\delta=4.87 \mathrm{ppm}$ was assigned to the protons attached to the carbon 'b' of the post-modified polymer (Figure 3a, bottom). Meanwhile, the peaks at $\delta=2.3-2.7 \mathrm{ppm}$ corresponding to the protons attached to the carbon $\mathbf{b}$ of PMVK disappeared after the oxidation (Figure 3a, top). These results indicated that the BV oxidation completely transformed the PMVK homopolymer into a PVAc homopolymer. The ${ }^{13} \mathrm{C}$ NMR spectra also provided additional evidence. In Figure $3 b$, the carbonyl signal of the ketone of PMVK $(\delta=210.2 \mathrm{ppm})$ disappeared completely after the oxidation while a carbonyl signal corresponding to an ester appeared at 170.4 ppm. In addition, the conversion of PMVK to PVAc was further confirmed by FT-IR.

The $M_{\mathrm{n}}$ and $\oslash$ of the resulting PVAc polymer $5 \mathrm{a}$ were obtained by SEC (Figure 4a). A slightly higher $M_{\mathrm{n}}$ and a similar $D$ were obtained after functionalization. The SEC traces indicated that our protocol successfully suppressed chain scission and cross-linking without affecting the MW and $\oslash$. Meanwhile, glass transition temperature $\left(T_{\mathrm{g}}\right)$ data was collected using differential scanning calorimetry (DSC) (Figure 4b). The $T_{\mathrm{g}}$ 

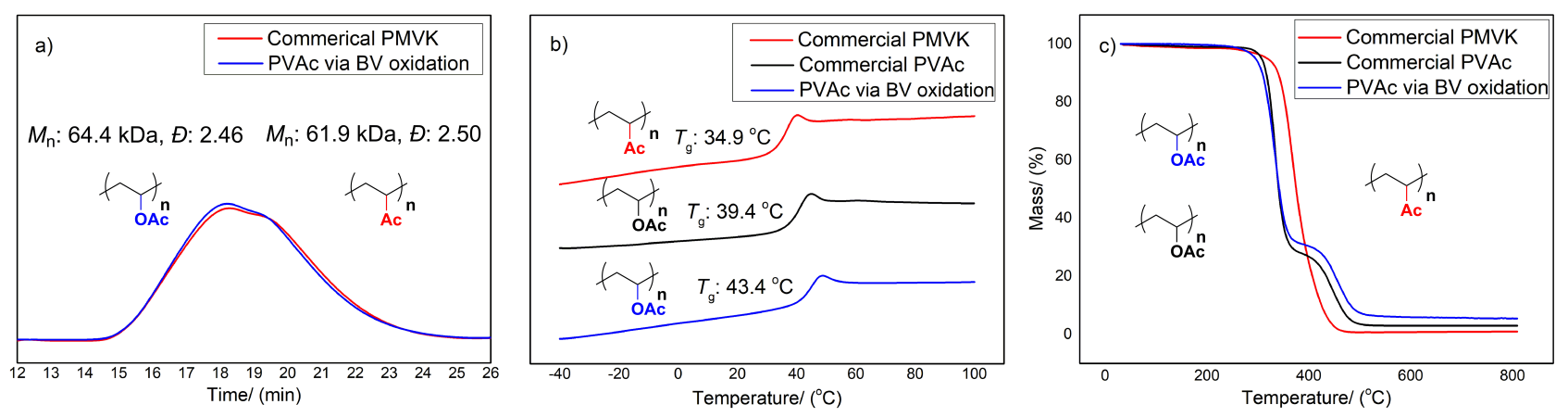

Figure 4. Characterization of PVAc 5a prepared by "exhaustive" BV post-modification of PMVK 3a. Comparison of (a) SEC, (b) DSC, and (c) TGA data of PMVK 3a, commercial PVAc and PVAc 5a obtained by BV oxidation.

of PVAc 5a obtained by the "exhaustive" BV oxidation was close to the $T_{\mathrm{g}}$ of the commercial PVAc sample. In contrast, the $T_{\mathrm{g}}$ of PMVK 3a was slightly lower. Furthermore, the thermogravimetric analysis (TGA) of this sample after oxidation displayed a twostaged curve, which is analogous to that of PVAc (Figure 4c). The initial degradation stage possibly relates to the elimination reaction of side groups while the second stage may relate to the degradation of the polymer backbone. As a comparison, the TGA of the original PMVK sample exhibited a one-stage curve.

"Exhaustive" BV Oxidation of Statistical Copolymers of MVK and Various Monomers. Although PVAc-derived copolymers are highly valuable, many PVAc copolymers, in particular those with more activated monomers (MAMs), are rarely accessed by conventional copolymerization approaches. ${ }^{[11]}$ Indeed, copolymerization cannot occur effectively since VAc and MAMs have disparate reactivity ratios. ${ }^{[11,12]}$ It is therefore highly desirable to develop a general method for the synthesis of poly(VAc-Co-MAM)s. It was much expected that our "exhaustive" BV post-modification would provide a general solution to the synthesis of poly(VAc-coMAM)s.

Firstly, we prepared a number of PMVK-co-PSs with differing MVK/St feed ratios (20/80, 50/50, and 80/20) under AIBN-initiated free radical polymerization. The reactivity ratios of MVK and St monomers $\left(r_{\mathrm{MVK}}=0.29, r_{\mathrm{St}}=0.35\right)^{[11]}$ indicate that PMVK-co-PS copolymers favor a statistical distribution. As expected, almost identical MVK/St ratios (28/72, 51/49 and $75 / 25$, respectively) were obtained in the resulting copolymers. Subsequently, the prepared PMVK-co-PS copolymers were functionalized under the BV protocol developed for the PMVK homopolymers. Unexpectedly, it was observed that the rates of BV oxidation for PMVK-Co-PS copolymers were higher than
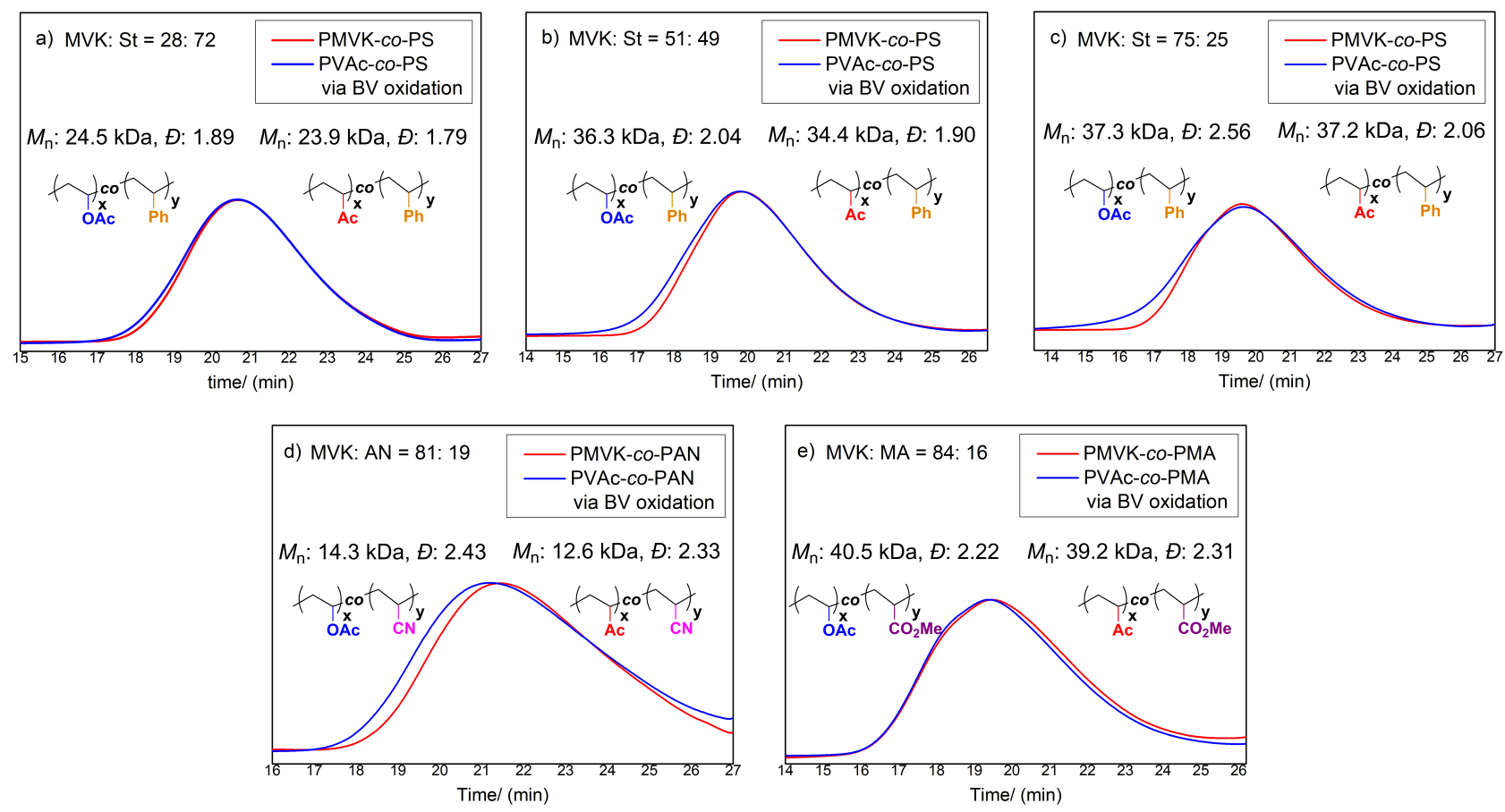

Figure 5. Synthesis of statistical copolymers of VAc and various monomers. SEC traces before and after the "exhaustive" BV oxidation of MVK statistical copolymers: (a) MVK/St (28/72) statistical copolymer 6a, (b) MVK/St (51/49) statistical copolymer 6b, (c) MVK/St (75/25) statistical copolymer 6c, (d) MVK/AN (81/19) statistical copolymer $6 \mathbf{d}$ and (e) MVK/MA (84/16) statistical copolymer 6 e. 
of the PMVK homopolymers. The enhanced rates appeared to be proportional to the percentages of styrene units within the PMVK-co-PSs. Following the same trend, the rates of chain scission and cross-linking also increased. Gratifyingly, with careful tuning of the reaction temperature, a set of "exhaustive" modification conditions for PMVK-Co-PS were achieved at $45{ }^{\circ} \mathrm{C}$ $(\mathrm{MVK} / \mathrm{St}=75 / 25), 40^{\circ} \mathrm{C}(\mathrm{MVK} / \mathrm{St}=51 / 49)$, and $35^{\circ} \mathrm{C}(\mathrm{MVK} / \mathrm{St}$ $=28 / 72$ ), respectively (Table S10). Under these optimized conditions, the $M_{n}$ and $\oplus$ were well controlled (Figure 5). The reactivity differences between PMVK homopolymers and PMVK-coPS copolymers can be tentatively attributed to the fact that phenyl groups are less electron-withdrawing than acetyl and acetoxy groups. Mechanistically, the reaction rate of BV oxidation can be affected by the electronic properties of the migrating group. Besides PVAc-co-PS, PVAc copolymers with other MAMs such as acrylonitrile (AN), and methyl acrylate (MA) were also successfully prepared. Under the optimized BV conditions of $50^{\circ} \mathrm{C}$ and 24 hours, PMVK-co-PAN and PMVK-co-PMA copolymers were fully converted to their corresponding PVAc-co-PAN and PVAcco-PMA copolymers, respectively. No obvious chain scission or cross-linking were observed in the SEC traces (Figure 5). All of the prepared statistical copolymers were thoroughly characterized. The "comonomer" ratios in the functionalized copolymers were consistent with the ratios of the starting copolymers (Table S10 and S12). Analysis of NMR spectral data (Figure S4-S8) confirmed that nearly all MVK units were converted to VAc units. FT-IR, DSC, and TGA data (Table S11 and S13) further supported the NMR spectra result.

"Exhaustive" BV Oxidation of Block Copolymers of MVK and Various Monomers. In addition to the synthesis of statistical copolymers, the synthesis of block copolymers of VAC and MAMs remains challenging as well. ${ }^{[20]}$ Encouraged by our success in the synthesis of statistical poly(VAc-co-MAM)s, we anticipated that our BV post-modification strategy could be expanded to the synthesis of PVAc block copolymers with MAMs. Firstly, we prepared a number of PMVKs and poly(MAM- $b$ MVK)s via RDRP techniques. Although the atom transfer radical polymerization (ATRP) of vinyl ketone monomers ${ }^{[15 a]}$ is presently inaccessible, Wooley's RAFT protocol[13f] offered us an effective method to synthesize narrow-dispersed PMVKs and PMVK block copolymers. Unfortunately, none of our established BV protocols for the statistical copolymers were compatible with the post-modification of narrow-dispersed PMVK homopolymers. Significant degradation of the polymer architecture occurred in all cases (Table S8). We speculated that the degradation was most likely caused by the end-group of the RAFT polymers. Indeed, this hypothesis was later verified by the fact that a RAFTderived polymer without a sulfur-based end group ${ }^{[21]}$ performed well using our BV protocol (Table S8).

Previous studies indicated that sulfonic acid might be generated by the oxidation of the sulfur-based end-groups in the RAFT polymers. ${ }^{[22]}$ Although $\mathrm{TMS}_{2} \mathrm{O}$ was unable to buffer the more acidic $-\mathrm{SO}_{3} \mathrm{H}$ group, we speculated that another additive might neutralize both $m$-chlorobenzoic acid and the additional sulfonic acid. Significant efforts at using common bases as a buffer met with little success since the $\mathrm{pH}$ of the reaction mixture changed dynamically. Indeed, a fluctuating $\mathrm{pH}$, no matter if higher or lower, could be detrimental to the post-modification. Gratifyingly, $t$-butyl carbamate and urea were later identified as the most effective additives after a laborious screening (Table 2 and Table S9). With a modified procedure using 0.2 equiv of urea as an additive, the PMVK homopolymer prepared by RAFT was nearly quantitatively transformed into a PVAc polymer without obvious chain scission or cross-linking. The structural integrity of the resulting PVAc polymer was verified by ${ }^{1} \mathrm{H}$ and ${ }^{13} \mathrm{C}$ NMR (Figure S3), and FT-IR. Furthermore, MALDI-TOF MS (matrix-assisted laser desorption/ionization time-of-flight mass spectroscopy) spectrum indicated a spacing of 86.1 is consistent with the MW of VAc repeating unit (Figure S13).

The modified BV protocol using the urea additive was further applied to the synthesis of PVAc block copolymers. Two di-block copolymers, poly(methyl methacrylate)-block-poly(methyl vinyl ketone) (PMMA- $b$-PMVK, $M_{\mathrm{n}}=14.0 \mathrm{kDa}, \mathrm{PMMA} / \mathrm{PMVK}=7.7 / 6.3$ ) and poly(ethyl acrylate)-block-poly(methyl vinyl ketone) (PEA- $b$ PMVK, $\left.M_{n}=13.9 \mathrm{kDa}, \mathrm{PEA} / \mathrm{PMVK}=8.6 / 5.3\right)$, and one "ABA"type tri-block copolymer, PMVK- $b$-PMA- $b$-PMVK $\left(M_{\mathrm{n}}=20.0 \mathrm{kDa}\right.$, PMVK/PMA/PMVK = 5.2/10.3/4.5), were synthesized by sequential RAFT polymerization. Subsequently, these three PMVK block copolymers were "exhaustively" functionalized by the urea-modified BV protocol, with PMMA- $b$-PVAc, PEA- $b$ PVAc, and PVAc- $b$-PMA- $b-P V A c$ being obtained without apparent degradation (Figure 6 and Figure S9-11). Notably, all

Table 2. Screening of BV oxidation conditions for RAFT homopolymer $\mathbf{3 b}$ in 1,2,4-trichlorobenzene. ${ }^{\text {[a] }}$

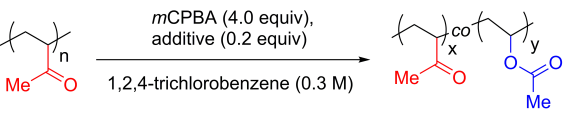

3b (RAFT polymer)

\begin{tabular}{|c|c|c|c|c|c|c|c|c|c|}
\hline Entry & Additive & $\begin{array}{c}\text { Conversion }^{[\mathrm{b}]} \\
(\%)\end{array}$ & $\begin{array}{c}M_{\mathrm{n}}{ }^{[\mathrm{c}]} \\
(\mathrm{kDa})\end{array}$ & $\oplus^{[c]}$ & Entry & Additive & $\begin{array}{c}\text { Conversion }^{[\mathrm{b}]} \\
(\%)\end{array}$ & $\begin{array}{c}M_{\mathrm{n}}^{[\mathrm{c}]} \\
(\mathrm{kDa})\end{array}$ & $\theta^{[\mathrm{c}]}$ \\
\hline $1^{[\mathrm{d}]}$ & hexamethyldisiloxane & n.a. & 2.1 & 1.63 & 9 & 1,3-diphenylurea & 69 & 4.3 & 1.39 \\
\hline $2^{[\mathrm{e}]}$ & n.a. & $>99$ & 4.4 & 1.43 & 10 & $p$-toluenesulfonamide & 98 & 3.3 & 1.35 \\
\hline 3 & triethylamine & 90 & 10.7 & 1.30 & 11 & urea & $>99$ & 13.5 & 1.15 \\
\hline 4 & $\mathrm{~N}, \mathrm{~N}$-diisopropylethylamine & 88 & 10.0 & 1.32 & 12 & thiourea & 98 & 11.8 & 1.21 \\
\hline 5 & 2,6-lutidine & 61 & 13.1 & 1.18 & 13 & ammonium carbamate & 87 & 6.9 & 1.37 \\
\hline 6 & $\mathrm{NH}_{4} \mathrm{HCO}_{3}$ & 96 & 9.4 & 1.29 & 14 & methyl carbamate & 98 & 3.6 & 1.38 \\
\hline 7 & $\mathrm{NH}_{4} \mathrm{OAC}$ & 83 & 6.9 & 1.34 & 15 & $t$-butyl carbamate & $>99$ & 13.0 & 1.16 \\
\hline 8 & benzamide & 99 & 10.7 & 1.25 & & & & & \\
\hline
\end{tabular}

[a] Standard conditions: RAFT homopolymer of MVK $3 \mathbf{b}$ (41.7 mg, containing $0.60 \mathrm{mmol}$ repeating units), $\mathrm{mCPBA}(2.40 \mathrm{mmol})$, additive (0.12 mmol), and solvent $(2.00 \mathrm{~mL})$ stirred at $50{ }^{\circ} \mathrm{C}$ for $24 \mathrm{~h}$ unless noted otherwise. The $\mathrm{Mn} / \oplus$ of PMVK was $12.5 \mathrm{kDa} / 1.07$. [b] Determined by ${ }^{1} \mathrm{H} \mathrm{NMR}$ of the crude products using 1,1,1,3-tetrachloropropane as an internal standard. [c] Determined by SEC (THF) analysis relative to polystyrene standards. [d] Hexamethyldisiloxane $(1.20 \mathrm{mmol})$ and dry $m$ CPBA were employed. [e] $m$ CPBA was used without drying. 

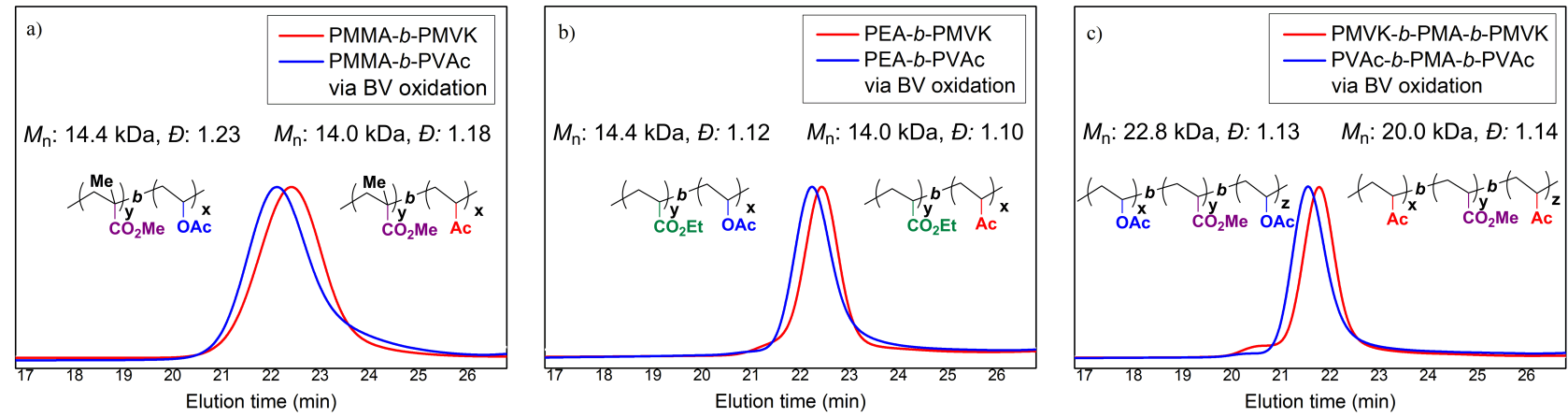

Figure 6. Synthesis of block copolymers of VAc and various monomers. SEC traces of (a) PMMA-b-PMVK di-block copolymer 7a, (b) PEA-b-PMVK di-block copolymer 7b and (c) PMVK-b-PMA-b-PMVK tri-block copolymer 7c before and after "exhaustive" BV oxidation.

of these copolymers were prepared in nearly identical conditions and the careful tuning of conditions for different MAMs were not required. This is distinct from the methods using switchable RAFT agent ${ }^{[23]}$ and dual RDRP agents ${ }^{[24]}$ where the choice of polymerization parameters and RDRP techniques were important. ${ }^{[20 a]}$

Preparation of PVAc-co-PS via One-pot Copolymerization/"Exhaustive" BV Post-modification. To streamline the preparation of poly(VAc-Co-MAM)s, we developed a one-pot copolymerization/"exhaustive" BV post-modification procedure (Figure 7). This convenient and scalable process allows us to synthesize PVAc-co-PS on a gram scale from MVK and St monomers directly without the purification of the PMVK-co-PS precursor. Noticeably, the use of 1,2,4-trichlorobenzene as a solvent for copolymerization was crucial for this process. Although the comprehensive properties of PVAc-co-PS $\mathbf{1 0}$ have not been investigated, this copolymer has exhibited an improved performance over the standard PVAc adhesive in our preliminary tests, especially when applied in aqueous conditions at elevated temperatures. ${ }^{[25]}$

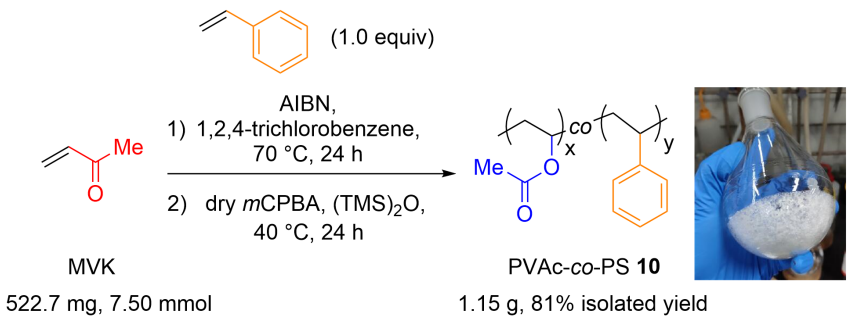

Figure 7. One-pot synthesis of PVAc-co-PS 10.

\section{Conclusion}

Baeyer-Villiger oxidation, a historically important transformation in small-molecule organic chemistry, has now emerged to be an efficient post-polymerization modification reaction for PMVK and its copolymers. Initial unsuccessful attempts on a small-molecule model led us to develop and reoptimize the BV oxidation directly on PMVK polymers. After the elucidation of multiple factors affecting reaction kinetics and side reactions, the precise tuning of reaction parameters allowed us to identify a set of tailor-made BV oxidation conditions which were able to "exhaustively" functionalize PMVK and its copolymers without obvious chain scission or cross-linking.

With this unique BV post-modification method in hand, we successfully prepared a broad array of poly(VAc-co-MAM) statistical copolymers that are rarely accessed by direct copolymerization. In addition, a number of PVAc di-block and triblock copolymers with narrow molecular weight distributions were synthesized using this post-modification method. A gramscale one-pot process was additionally developed to demonstrate the potential of a scalable production of such copolymers. The material properties and potential applications of the prepared poly(VAc-Co-MAM) copolymers will be reported in due course.

In addition, we recognize that re-optimizing a reaction on polymer substrates could be a valuable approach for the development of other post-polymerization modification reactions, particularly when the direct translation of small-molecule protocols failed. Post-modifications of other commodity polymers such as poly(acrylic acid) and polyketones using this approach are currently being investigated in our laboratory.

\section{Acknowledgements}

Financial support from the National Natural Science Foundation of China (No. 21502241), the Natural Science Foundation of Guangdong Province (No. 2016A030313290), and Sun Yat-sen University is gratefully acknowledged. L.L. thanks Prof. Daniel Seidel (University of Florida), Prof. Seth B. Herzon (Yale University), Prof. Julian Zhu (Université de Montréal), Prof. Yongguang Jia (South China University of Technology), Prof. Hanchu Huang (Sun Yat-sen University), and Pyh Li for helpful discussions and support.

\section{Conflict of interest}

Sun Yat-sen University has filed a patent application.

Keywords: Post-polymerization modification • Baeyer-Villiger oxidation - Reactivity ratios - PVAc copolymers • Block polymers 
[1] Selected reviews in recent literature: a) C. M. Plummer, L. Li, Y. Chen, Polym. Chem. 2020, 11, 6862-6872; b) J. B. Williamson, S. E. Lewis, R. R. Johnson III, I. M. Manning, F. A. Leibfarth, Angew. Chem. Int. Ed. 2019, 58, 8654-8668; Angew. Chem. 2019, 131, 8746-8761; c) E. Blasco, M. B. Sims, A. S. Goldmann, B. S. Sumerlin, C. Barner-Kowollik, Macromolecules 2017, 50, 5215-5252; d) J. Romulus, J. T. Henssler, M. Weck, Macromolecules 2014, 47, 5437-5449; e) K. A. Günay, P. Theato, H.-A. Klok, J. Polym. Sci., Part A: Polym. Chem. 2013, 51, 128; f) M. A. Gauthier, M. I. Gibson, H.-A. Klok, Angew. Chem. Int. Ed. 2009, 48, 48-58; Angew. Chem. 2009, 121, 50-60; g) N. K. Boaen, M. A. Hillmyer, Chem. Soc. Rev. 2005, 34, 267-275.

[2] Selected reviews on click chemistry: a) J. Dong, L. Krasnova, M. G. Finn, K. B. Sharpless, Angew. Chem. Int. Ed. 2014, 53, 9430-9448; Angew. Chem. 2014, 126, 9584-9603; b) J. E. Moses, A. D. Moorhouse, Chem. Soc. Rev. 2007, 36, 1249-1262; c) W. H. Binder, R. Sachsenhofer, Macromol. Rapid Commun. 2007, 28, 15-54; d) H. Kolb, M. G. Finn, K. B. Sharpless, Angew. Chem. Int. Ed. 2001, 40, 2004-2021; Angew. Chem. 2001, 113, 2056-2075.

[3] Selected reviews on multicomponent reactions: a) M. A. R. Meier, R. Hu, B. Tang, Macromol. Rapid Commun. 2021, 42, 2100104; b) R. Kakuchi, Polym. J. 2019, 51, 945-953.

[4] a) J. Lee, A. J. Kalin, T. Yuan, M. Al-Hashimi, L. Fang, Chem. Sci. 2017, 8, 2503-2521; b) R. Kakuchi, P. Theato, "Post-polymerization Modifications via Active Esters" in Functional Polymers by Post-Polymerization Modification: Concepts, Guidelines, and Applications, 1st ed., (Eds.: P. Theato, H.-A. Klok), Wiley-VCH, Weinheim, 2013, pp. 45-64.

[5] K. Pahnke, J. Brandt, G. Gryn'ova, C. Y. Lin, O. Altintas, F. G. Schmidt, A. Lederer, M. L. Coote, C. Barner-Kowollik, Angew. Chem. Int. Ed. 2016, 55, 1514-1518; Angew. Chem. 2016, 128, 1537-1541.

[6] Selected examples in recent literature: a) P. Jung, A. D. Ziegler, J. Blankenburg, H. Frey, Angew. Chem. Int. Ed. 2019, 58, 12883-12886; Angew. Chem. 2019, 131, 13015-13018; b) Y. M. Wu, T. M. Swager, J. Am. Chem. Soc. 2019, 141, 12498-12501; c) C. Jeon, D. W. Kim, S. Chang, J. G. Kim, M. Seo, ACS Macro Lett. 2019, 8, 1172-1178; d) M. B. Larsen, S. Wang, M. A. Hillmyer, J. Am. Chem. Soc. 2018, 140, 11911-11915.

[7] Selected examples on oxidative post-polymerization modifications: a) L. Chen, K. G. Malollari, A. Uliana, J. F. Hartwig, J. Am. Chem. Soc. 2021, 143, 4531-4535; b) L. Chen, K. G. Malollari, A. Uliana, D. Sanchez, P. B. Messersmith, J. F. Hartwig, Chem 2021, 7, 137-145; c) Y. Dong, Z. Wang, C. Li, Nat. Commun. 2017, 8, 277; d) A. Bunescu, S. Lee, Q. Li, J. F. Hartwig, ACS Cent. Sci. 2017, 3, 895-903; e) N. K. Boaen, M. A. Hillmyer, Macromolecules 2003, 36, 7027-7034.

[8] H. L. van de Wouw, J. Y. Lee, E. C. Awuyah, R. S. Klausen, Angew. Chem. Int. Ed. 2018, 57, 1673-1677; Angew. Chem. 2018, 130, 16891693

[9] T. Nishikawa, M. Ouchi, Angew. Chem. Int. Ed. 2019, 58, 12435-12439; Angew. Chem. 2019, 131, 12565-12569.

[10] a) T. Chinnusamy, K. Feeney, C. G. Watson, D. Leonori, V. K. Aggarwal, "Oxidation of Carbon-Boron Bonds" in Comprehensive Organic Synthesis II, 2nd ed., (Eds.: P. Knochel), Elsevier, Amsterdam, 2014, pp. 692-718; b) H. C. Brown, C. Synder, B. C. Subba Rao, G. Zweifel, Tetrahedron 1986, 42, 5505-5510; c) G. Zweifel, H. C. Brown, Org. React. 1963, 13, 1-54.

[11] Principles of Polymerization, 4th ed., (Eds.: G. Odian), John Wiley \& Sons, New York, 2004.

[12] a) F. R. Mayo, F. M. Lewis, C. Walling, J. Am. Chem. Soc. 1948, 70, 1529-1533; b) F. R. Mayo, C. Walling, F. M. Lewis, W. F. Hulse, J. Am. Chem. Soc. 1948, 70, 1523-1525; c) F. R. Mayo, F. M. Lewis, J. Am. Chem. Soc. 1944, 66, 1594-1601.

[13] a) I. Lee, E. H. Discekici, A. Anastasaki, J. R. de Alaniz, C. J. Hawker, Polym. Chem. 2017, 8, 3351-3356; b) J. A. Reeves, M. L. Allegrezza,
D. Konkolewicz, Macromol. Rapid Commun. 2017, 38, 1600623; c) Y. Zhao, X. Liu, Y. Liu, Z. Wu, X. Zhao, X. Fu, Chem. Commun. 2016, 52, 12092-12095; d) J. A. M. Hepperle, H. Luftmann, A. Studer, J. Polym. Sci., Part A: Polym. Chem. 2012, 50, 2150-2160; e) K. Uehara, C. B. Wagner, T. Vogler, H. Luftmann, A. Studer, Angew. Chem. Int. Ed. 2010, 49, 3073-3076; Angew. Chem. 2010, 122, 3137-3141; f) C. Cheng, G. Sun, E. Khoshdel, K. L. Wooley, J. Am. Chem. Soc. 2007, 129, 1008610087.

[14] Selected reviews on Baeyer-Villiger oxidation: a) L. Zhou, L. Lin, X. Liu, $X$. Feng, "Baeyer-Villiger (BV) Oxidation/Rearrangement in Organic Synthesis" in Molecular Rearrangements in Organic Synthesis, 1st ed., (Eds.: C. M. Rojas), John Wiley \& Sons, Hoboken, 2016, pp. 35-57; b) G. J. ten Brink, I. W. C. E. Arends, R. A. Sheldon, Chem. Rev. 2004, 104, 4105-4123; c) M. Renz, B. Meunier, Eur. J. Org. Chem. 1999, 737-750; d) G. R. Krow, Org. React. 1993, 43, 251-798.

[15] a) A. Mittal, S. Sivaram, D. Baskaran, Macromolecules 2006, 39, 55555558; b) N. Kosaka, T. Hiyama, K. Nozaki, Macromolecules 2004, 37, 4484-4487; c) A. W. P. Jarvie, N. Overton, C. B. S. Pourçain, J. Chem. Soc., Perkin Trans. 1, 1999, 15, 2171-2176.

[16] a) J. W. Krumpfer, E. Giebel, E. Frank, A. Muller, L. Ackermann, C. N. Tironi, G. Mourgas, J. Unold, M. Klapper, M. R. Buchmeiser, K. Mullen, Chem. Mater. 2017, 29, 780-788; b) J. N. Hay, Makromol. Chem. 1963 67, 31-41; c) C. S. Marvel, C. L. Levesque, J. Am. Chem. Soc. 1938, 60, 280-284.

[17] a) M. C. Celina, Polym. Degrad. Stab. 2013, 98, 2419-2429; b) T. Q. Nguyen, "Polymer Degradation and Stabilization" in Handbook of Polymer Reaction Engineering, (Eds.: T. Meyer, J. Keurentjes), Wiley-VCH, Weinheim, 2005, pp. 757-831.

[18] B. Schweitzer-Chaput, T. Kurten, M. Klussmann, Angew. Chem. Int. Ed. 2015, 54, 11848-11851; Angew. Chem. 2015, 127, 12015-12018.

[19] Our preliminary studies indicated that BV oxidations of other poly(vinyl ketones) were less regioselective than that of PMVK.

[20] Selected reviews in recent literature: a) S. Pearson, C. S. Thomas, R. Guerrero-Santos, F. D'Agosto, Polym. Chem. 2017, 8, 4916-4946; b) S. Harrisson, X. Liu, J. Ollagnier, O. Coutelier, J. Marty, M. Destarac, Polymers 2014, 6, 1437-1488; c) L. E. N. Allan, M. R. Perry, M. P. Shaver, Prog. Polym. Sci. 2012, 37, 127-156; d) H. Tang, M. Radosz, Y. Shen, "Controlled/"Living" Radical Polymerization of Vinyl Acetate" in Controlled/Living Radical Polymerization: Progress in ATRP, (Eds.: K. Matyjaszewski), ACS Symposium Series 1023, Washington, DC, 2009; pp. 139-157; e) Handbook of RAFT Polymerization, (Eds.: C. Barner-Kowollik), Wiley-VCH, Weinheim, 2008.

[21] a) E. H. Discekici, S. L. Shankel, A. Anastasaki, B. Oschmann, I. Lee, J. Niu, A. J. McGrath, P. G. Clark, D. Laitar, J. Read de Alaniz, C. J. Hawker, D. J. Lunn, Chem. Commun. 2017, 53, 1888-1891; b) H. Willcock, R. K. O'Reilly, Polym. Chem. 2010, 1, 149-157; c) M. Chen, G. Moad, E. Rizzardo, J. Polym. Sci., Part A: Polym. Chem. 2009, 47, 6704-6714; d) S. Perrier, P. Takolpuckdee, C. A. Mars, Macromolecules 2005, 38, 2033-2036.

[22] L. Feng, K. A. Cavicchi, B. C. Katzenmeyer, C. Wesdemiotis, J. Polym. Sci., Part A: Polym. Chem. 2011, 49, 5100-5108.

[23] M. Benaglia, J. Chiefari, Y. K. Chong, G. Moad, E. Rizzardo, S. H. Thang, J. Am. Chem. Soc. 2009, 131, 6914-6915.

[24] a) R. Nicolay, Y. Kwak, K. Matyjaszewski, Chem. Commun. 2008, 42, 5336-5338; b) Y. Tong, Y. Dong, F. Du, Z. Li, Macromolecules 2008, 41, 7339-7346.

[25] Our preliminary studies indicated that the ability of PVAC-Co-PS 10 to adhere glass maintained after at $24 \mathrm{~h}$ in hot water $\left(60^{\circ} \mathrm{C}\right)$ while PVAC lost its adhesive strength in less than $5 \mathrm{~min}$. 